\title{
Double critical coupled ring resonator-based add-drop filters
}

\author{
Mahdi Bahadoran ${ }^{1} \cdot$ Iraj Sadegh Amiri ${ }^{2,3}$
}

Received: 20 May 2019 / Accepted: 7 August 2019 / Published online: 15 August 2019

(c) The Author(s) 2019

\begin{abstract}
In this paper, we introduced double-critical coupling condition (DCCC) for lossy mode couplers of the add-drop resonator to achieve the desirable and tunable signals required for optical communication and photonics sensors applications. The performance of add-drop resonator under double-critical condition is simulated and analyzed. Some equations were derived for optical parameters including full width at half maximum (FWHM), the out-of-band rejection ratio (OBRR), quality factor and the crosstalk of the add-drop resonator, and the output signals were examined as a function of DCCC. Based on double-critical condition, the OBRR values larger than $40 \mathrm{~dB}$, the crosstalk larger than $50 \mathrm{~dB}$, highest quality factor of 9000 and the FWHM as small as $0.17 \mathrm{~nm}$ were realized in silicon add-drop resonator, which are quite compatible with the reported experimental data. Double-critical condition shows that the lossless coupling is not enough condition to acquire high-quality filtered signal with a large crosstalk and in presence of coupling losses, the DCCC can determine the optimum relation between the strength of coupling coefficients and the coupling losses.
\end{abstract}

Keywords Add-drop filter · Double-critical coupling condition $\cdot$ Microring resonator $\cdot$ Calibration signals $\cdot$ Tuning FWHM · Crosstalk

\section{Introduction}

Optical communication has been receiving considerable attention due to the growing demand for high-speed internet, multimedia communication and data-dense applications [1]. The capacity limitation of electro-optical networks gives rise to the emergence of all optical networks, which are capable to support various optical add-drop multiplexers with a vast bandwidth. Integrated ring resonators have found a number of usages in integrated optics due to their compact size, low cost of fabrication and their ability to integrate with on chip circuits. Today, micro-size resonators are practical devices

Iraj Sadegh Amiri

irajsadeghamiri@tdtu.edu.vn

Mahdi Bahadoran

bahadoran@sutech.ac.ir

1 Department of Physics, Shiraz University of Technology, Shiraz, Fars 31371555, Iran

2 Computational Optics Research Group, Advanced Institute of Materials Science, Ton Duc Thang University, District 7, Ho Chi Minh City 700000, Vietnam

3 Faculty of Applied Sciences, Ton Duc Thang University, District 7, Ho Chi Minh City 700000, Vietnam for switching [2-4], multiplexing, demultiplexing [5] and wavelength filtering [6, 7], modulation applications [8], wide band antenna $[9,10]$, modulator and logic gate in optical signal processing $[11,12]$, and sensitive sensors and biosensors [13-15]. One of the primary applications of ring resonators is their application as an add-drop notch type filter in optical networks. Based on the coupler position and type, different layouts of add-drop resonators have been reported for filtering signals, which include the symmetrically coupled [16] and asymmetrically coupled add-drop resonator [17] and one-stage parallel-coupled add-drop resonators [18]. Applying multiple stage resonators can also increase the quality of the output signals and improve the crosstalk between channels [19]. Generally, the crosstalk in optical filters emerges due to the miss match in channel wavelengths [20]. One of the approaches in reducing the mismatch in the channel wavelengths is considering the resonance condition in the ring resonator and its effect on the free spectral range. Indeed, the resonant mode number, the perimeter of the ring resonator, the group refractive index of ring's waveguide and the resonance wavelength are determining factors [21]. These parameters together with critical condition need to be considered in the fabrication and modeling part. It is reported that the crosstalk can be controlled by applying 
vertical coupling in add-drop resonator [22], and it can be reduced via the scheme of a pair of the parallel coupled of critical resonators [23]. However, a mathematical relation is required to tune the crosstalk. Totally, to use ring resonator as an optical filter or an optical sensing device, the output signals need to be tunable. Since traveling of signals via different couplers can combine the fields and cause the interference, adjusting the parameters associated with couplers contribute to achieve high quality signals.

In this work, we introduced a simultaneous two-lossymode critical coupling condition to achieve the optimum transmission from add-drop resonator. Based on this condition, the through and drop port signals from add-drop resonator were tuned to achieve the desirable signals. The quality of signals was examined in terms of the full width at half maximum (FWHM), quality factor, out-of-band rejection ratio and the crosstalk and compared with data from fabricated silicon add-drop filter. The introduced doublecritical condition is applicable in optical filtering, photonics sensors, terrestrial microwave, Wi-Fi devices and WDM technologies.

\section{Theoretical background}

The Z-transform has been employed for analyzing the discrete time systems and manipulating the discrete data sequences extensively in the fields of digital filtering and signal processing [24, 25], applied mathematics [26], economics [27] and control theory [28]. The signal flow graph (SFG) method takes advantage of Mason's rule [29, 30] and the Z-transform [31,32] to calculate optical transfer function of photonic devices. The SFG method can be interpreted as a transformation of either the method of successive substitution of simultaneous equations or the transfer matrix method to a topological approach. Based on the Mason's rule, the transfer function from input node $E_{\text {in }}$ to output node $E_{\text {out }}$ in a SFG diagram is given by

$\mathrm{OTF}=\frac{E_{\text {out }}}{E_{\text {in }}}=\frac{\sum_{m=1}^{N} T_{m} \Delta_{m}}{1-\sum_{i} L_{i}+\cdots}$

where OTF is the input to output signals' ratio, $T_{m}$ displays the gain of the $m$ th frontward path from input to output port, $N$ shows the total number of frontward tracks from input to output ports, and $L_{i}$ is the transmittance gain of each loop [33]. Schematic design of a ring resonator with two bus waveguides and a couple of $2 \times 2$ optical couplers known as add-drop ring resonator (ADR) is shown in Fig. 1. The coupling power $k_{1}$ and $k_{2}$ specified by the gap and the length that the waveguides were coupled. Using the Mason rule for

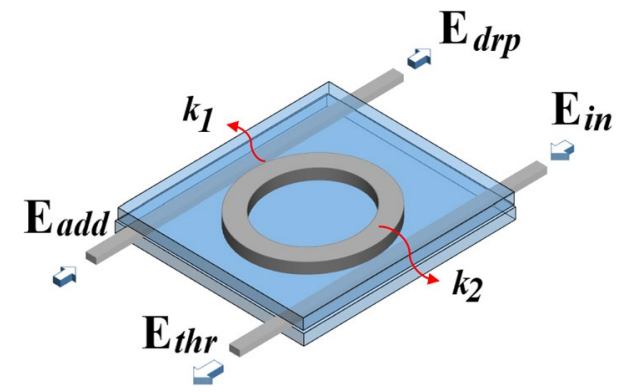

Fig. 1 Schematic diagram of single add-drop resonator

ADR layout in Fig. 1, the optical transfer function for the drop port of add-drop resonator becomes:

$$
\begin{aligned}
& \mathrm{OTF}_{\mathrm{drp}}=\frac{E_{\mathrm{drp}}}{E_{\mathrm{in}}} \\
& \quad=\frac{-\sqrt{\left(1-\gamma_{1}\right) k_{1}} \sqrt{\left(1-\gamma_{2}\right) k_{2}} e^{-\left(\alpha L / 4+i \pi n_{g} L / \lambda\right)}}{1-\sqrt{\left(1-\gamma_{1}\right)\left(1-k_{1}\right)} \sqrt{\left(1-\gamma_{2}\right)\left(1-k_{2}\right)} e^{-\left(\alpha L / 2+i 2 \pi n_{g} L / \lambda\right)}}
\end{aligned}
$$

where $k_{m}(m=1,2)$ is the coupling strength for each coupler, the amount of loss in each coupling region is given by $\gamma_{m}(m=1,2)$, the waveguide loss denoted by $\alpha, \mathrm{L}$ is the circumference of the ring resonator, $n_{g}$ denotes the group refractive index of the ring's waveguide, and $\lambda$ shows the wavelength of the input light. The exponential term in OTF $\xi=e^{-\left(\alpha L / 2+i 2 \pi n_{g} L / \lambda\right)}$ is known as loss-unit delay parameter [34-37]. The normalized intensity relation for the outputto-input port of resonating system can be obtained from the scalar product of OTF by its complex conjugates for each port. The normalized intensity of the drop port of ADR is calculated as

$$
\begin{aligned}
I_{\mathrm{drp}}= & \frac{\left(1-\gamma_{1}\right)\left(1-\gamma_{2}\right) k_{1} k_{2} e^{-\alpha L}}{\left[1-\sqrt{\left(1-\gamma_{1}\right)\left(1-k_{1}\right)} \sqrt{\left(1-\gamma_{2}\right)\left(1-k_{2}\right)} e^{-0.5 \alpha L}\right]^{2}} \\
& +4 \sqrt{\left(1-\gamma_{1}\right)\left(1-k_{1}\right)} \sqrt{\left(1-\gamma_{2}\right)\left(1-k_{2}\right)} e^{-0.5 \alpha L} \sin ^{2}\left(\frac{\varphi}{2}\right)
\end{aligned}
$$

here $\varphi$ represents the phase constant that emerged in each round-trip traveling of light via the ADR system. The optical transfer function for through port of ADR for lossy mode couplers is

$$
\begin{aligned}
& \mathrm{OTF}_{\mathrm{thr}}=\frac{E_{\mathrm{thr}}}{E_{\mathrm{in}}} \\
& \quad=\frac{\sqrt{\left(1-\gamma_{1}\right)\left(1-k_{1}\right)}-\sqrt{\left(1-\gamma_{2}\right)\left(1-k_{2}\right)} e^{-\left(\alpha L / 2+i 2 \pi n_{g} L / \lambda\right)}}{1-\sqrt{\left(1-\gamma_{1}\right)\left(1-k_{1}\right)} \sqrt{\left(1-\gamma_{2}\right)\left(1-k_{2}\right)} e^{-\left(\alpha L / 2+i 2 \pi n_{g} L / \lambda\right)}}
\end{aligned}
$$

The normalized intensity of through port of ADR is 


$$
\begin{aligned}
I_{\mathrm{thr}}=1- & \frac{\left(1-\gamma_{1}\right) k_{1}\left[1-e^{-\alpha L}\left(1-\gamma_{2}\right)\left(1-k_{2}\right)\right]}{\left[1-\sqrt{\left(1-\gamma_{1}\right)\left(1-k_{1}\right)} \sqrt{\left(1-\gamma_{2}\right)\left(1-k_{2}\right)} e^{-0.5 \alpha L}\right]^{2}} \\
& +4 \sqrt{\left(1-\gamma_{1}\right)\left(1-k_{1}\right)} \sqrt{\left(1-\gamma_{2}\right)\left(1-k_{2}\right)} e^{-0.5 \alpha L} \sin ^{2}\left(\frac{\varphi}{2}\right)
\end{aligned}
$$

Another significant parameter in characterizing the output signal quality from optical devices is the FWHM. According to Eqs. (3) and (5), the width of output signals at half of the maximum amplitude can be changed by the phase constant of $\varphi=4 \pi^{2} f n_{g} R / c$. The maximum intensity at the drop port of the ADR corresponds to $\varphi=2 m \pi(m=0,1,2, \ldots)$; thus by considering the drop port intensity equal to half of its maximum value, the phase constant becomes

$$
\begin{aligned}
\varphi & =\cos ^{-1}\left\{\begin{array}{c}
{\left[4 \sqrt{\left(1-\gamma_{1}\right)\left(1-\gamma_{2}\right)\left(1-k_{1}\right)\left(1-k_{2}\right)} e^{-\alpha L}+\right.} \\
\frac{\left.-\left(1-\gamma_{1}\right)\left(1-\gamma_{2}\right)\left(1-k_{1}\right)\left(1-k_{2}\right) e^{-\alpha L}-1\right]}{2 \sqrt{\left(1-\gamma_{1}\right)\left(1-\gamma_{2}\right)\left(1-k_{1}\right)\left(1-k_{2}\right)} e^{-\alpha L}}
\end{array}\right\} \\
& =\cos ^{-1}(\psi)
\end{aligned}
$$

Suppose that the difference in the round-trip phases occurs between the point of the maximum intensity and the point at half of the maximum intensity, thus the difference in the round-trip phases can be written as [38]

$\Delta \varphi=\varphi_{1}-\varphi_{2}=2 \cos ^{-1}(\psi)$

In terms of the frequency, the round-trip phases can be written by $\Delta \varphi=4 \pi^{2} n_{g} R \Delta f / c$. This equation can be expressed in terms of the wavelength by $\Delta \varphi=4 \pi^{2} n_{g} R \Delta \lambda / \lambda^{2}$. By considering Eq. (7), the FWHM of add-drop resonator, $\Delta \lambda_{\mathrm{FWHM}}$, is calculated as

$\Delta \lambda_{\mathrm{FWHM}}=\frac{\lambda^{2} \cos ^{-1}(\psi)}{2 \pi^{2} n_{g} R}$

The other quantity for characterization of the quality of the output signals is the quality factor, which shows the stored energy to the energy loss per cycle. In the context of resonators, the ability of the waveguide to confine the field is described by the quality factor, which shows a measure of the sharpness of the resonance peaks. It is defined as the ratio of the resonance wavelength $\lambda_{0}$ to the FWHM [39]. Based on Eq. (8), the quality factor for ADR can be determined as

$Q$ factor $=\frac{\lambda_{o}}{\Delta \lambda_{\mathrm{FWHM}}}=\frac{\pi n_{g} L}{\lambda \cos ^{-1}(\psi)}$

Determining minimum and maximum intensities contributes to specify the out-of-band rejection ratio, $O B R R$, as

$\mathrm{OBRR}=10 \log _{10}\left(\frac{I_{\mathrm{drop}}^{\max }}{I_{\mathrm{drop}}^{\min }}\right)$
The difference in channels wavelengths causes a crosstalk between optical channels in optical filters. The overlapping areas between relative through/input port transmission and relative drop/input port transmission known as crosstalk areas can be calculated by

Crosstalk $=10 \log _{10}\left(\frac{I_{\mathrm{drop}}^{\max }}{I_{\mathrm{thr}}^{\min }}\right)$

\section{Result and discussion}

In order to achieve the desirable resonance peaks for practical use in optical communication and photonics sensor applications, it is required to achieve the sharpest resonance peaks with narrowest FWHM and the maximum OBRR. To this end, some ring's parameters like coupling coefficients, coupling losses and waveguide loss are determining factors, which are required to be optimized. In this work, the experimental data from the silicon microring resonator with radius as small as $R=1.5 \mu \mathrm{m}$ and the group refractive index of $n_{g}=4.2$ [40] are used for simulation. As simulated in Fig. 2, the variation of coupling coefficients between bus waveguide and the ring waveguide in ADR can affect the intensities at the drop and through ports, the OBRR and the FWHM of output signals. Simulated results in Fig. 2a, b show that the intensity in the drop port of ADR increased by increasing the values of coupling coefficients; however, an inverse reaction can be realized for the output signals from the through port. A trade-off behavior can be observed between simultaneous responses of the through and drop ports. Based on the result in Fig. 2c, the out-of-band rejection ratio larger than $20 \mathrm{~dB}$ can be achieved by power coupling values $<0.3$. The OBRR values larger than $40 \mathrm{~dB}$ can be realized by conducting $10 \%$ of input signals into the ring's waveguide. Figure $2 \mathrm{~d}$ shows that the sharp and narrow signals with desired FWHM $<2$ nm can also be measured for the coupling coefficients $<0.1$. As demonstrated in Fig. 2d, for the specific relation in coupling coefficients, the FWHM is reached to a plateau with a constant value as large as $60.85 \mathrm{~nm}$, which is equal to the FSR of the applied ADR. It means the coupling coefficients $<0.1$ can again fulfill our expectations to achieve the resonance peaks with narrow FWHM and high OBRR, which are desirable for sensing applications.

\section{Lossy mode critical coupling}

The relative phases of the signals through the recombination process determine whether they interfere destructively or constructively. Sharp and narrow resonance peaks are required for optical communications and sensor applications. To obtain a mathematical relation between couplers, it is supposed that light transmission via the through port of 

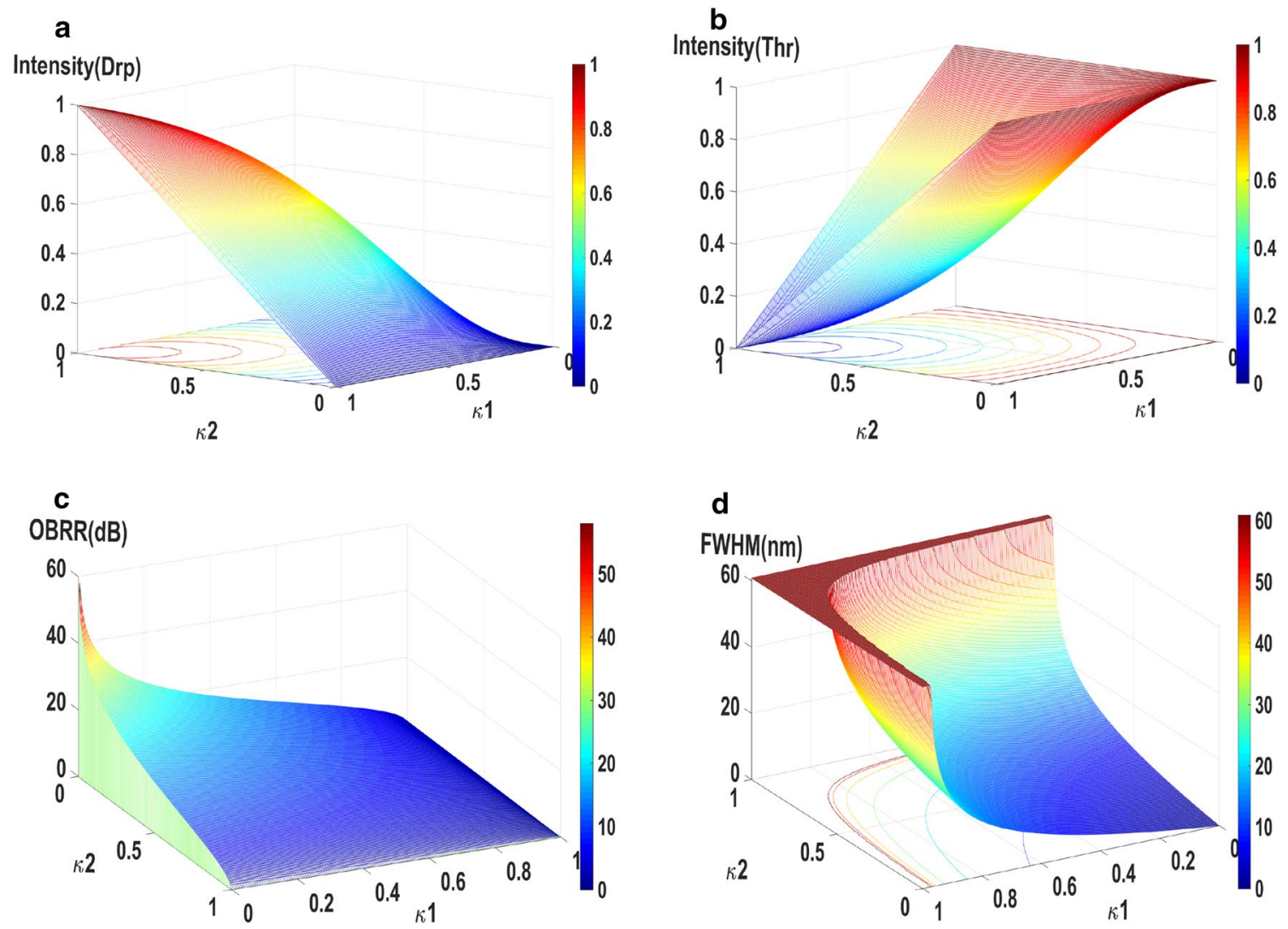

Fig. 2 The effect of changing coupling coefficients of the add-drop resonators on the a drop port intensity. b Through port intensity. $\mathbf{c}$ Out-ofband rejection ratio (OBRR). d Full width at half maximum (FWHM)

ADR reaches to its minimum value, $\mathrm{OTF}_{\mathrm{Thr}}(\phi=2 m \pi)=0$; in the other words, the maximum transmission can be realized at the drop port of ADR, $\operatorname{OTF}_{\mathrm{Drp}}(\phi=2 m \pi)=1$. Two critical coupling coefficients are calculated from $\mathrm{OTF}_{\mathrm{Thr}}(\phi=2 m \pi)=0$ and $\mathrm{OTF}_{\mathrm{Drp}}(\phi=2 m \pi)=1$ as follows:

$k_{C t}^{H \text { th }}=1-\frac{1-k_{1}}{\left(1-\gamma_{1}\right)\left(1-\gamma_{2}\right)}$

and

$$
\begin{aligned}
\left\{ \pm 4 \sqrt{\left[\left(1-\gamma_{1}\right)^{3}\left(1-\gamma_{2}\right)^{3}-\left(1-\gamma_{1}\right)^{2}\left(1-\gamma_{2}\right)^{2}\right]\left(k_{1}-k_{1}^{2}\right)}\right. & \left.-\left(1-\gamma_{1}\right)\left(1-\gamma_{2}\right)\left[2\left(1-\gamma_{1}\right)\left(1-\gamma_{2}\right)\left(k_{1}-1\right)-4 k_{1}+2\right]\right\} \\
k_{C t}^{H \mathrm{drp}}= & \frac{\left.-\gamma_{1}\right)^{2}\left(1-\gamma_{2}\right)^{2}}{2(13)}
\end{aligned}
$$

where $k_{1}$ shows a power coupling coefficient for one of the couplers in add-drop resonator system and $k_{C t}$ represents the value of the second coupling confident. The coupling losses in each coupling region of ADR are shown by $\gamma_{1}$ and $\gamma_{2}$. For simplicity, the first term of Taylor expansion is considered for the exponential terms in OTF $\xi=e^{-\left(\alpha L / 2+i 2 \pi n_{g} L / \lambda\right)} \approx 1$. Based on Eqs. (12) and (13), both critical coupling conditions are considered and the critical coupling values were plotted for add-drop resonator with lossy mode couplers. It is supposed that both couplers have equal coupling losses and the amount of light coupled into the ring waveguide can change from 0 to 100 percent. Figure $3 \mathrm{a}$, b shows the critical coupling values for zero through port output Eq. (12) and all-pass light via the drop port Eq. (13), respectively. Both critical conditions are demonstrated in Fig. 3c. Both critical conditions lead to the same line for lossless couplers $\left(\gamma_{1}=\gamma_{2}=0\right)$, which is the intercept line of two surfaces. The view of Fig. $3 \mathrm{c}$ from the $z$ direction is given in Fig. 3d. In order to find the most effective over coupling areas on the light transmission of add-drop resonator, 12 points are selected from both critical surfaces as addressed from $\mathrm{A}$ to $\mathrm{L}$ in Fig. 3d. The area pinned by B, E, H and L is located on the common intercept line, which is fulfilled over DCCC. The optical transmission of the add-drop resonator against wavelength was plotted for selected points (A-L) as shown in Fig. 4. The coupling coefficients and coupling losses of these selected points are given in Table 1. The points on the intercept line at Fig. 3c, d (B, E, H and L) represent the lossless coupling that fulfills the double-critical coupling condition. However, increase in coupling losses $\left(\gamma_{1}\right.$ 
Fig. 3 All-inclusive critical coupling values for lossy mode couplers of ADR. Critical coupling surface for $\mathbf{a ~ O T F}_{\text {Thr }}(\phi=2 m \pi)=0$. b $\operatorname{OTF}_{\operatorname{Drp}}(\phi=2 m \pi)=1$. c Double-critical coupling condition $\mathrm{OTF}_{\mathrm{Thr}}=0 \& \mathrm{OTF}_{\mathrm{drp}}=1$ and $\mathbf{d}$ double-critical coupling condition from $\mathrm{z}$ direction view (2D)
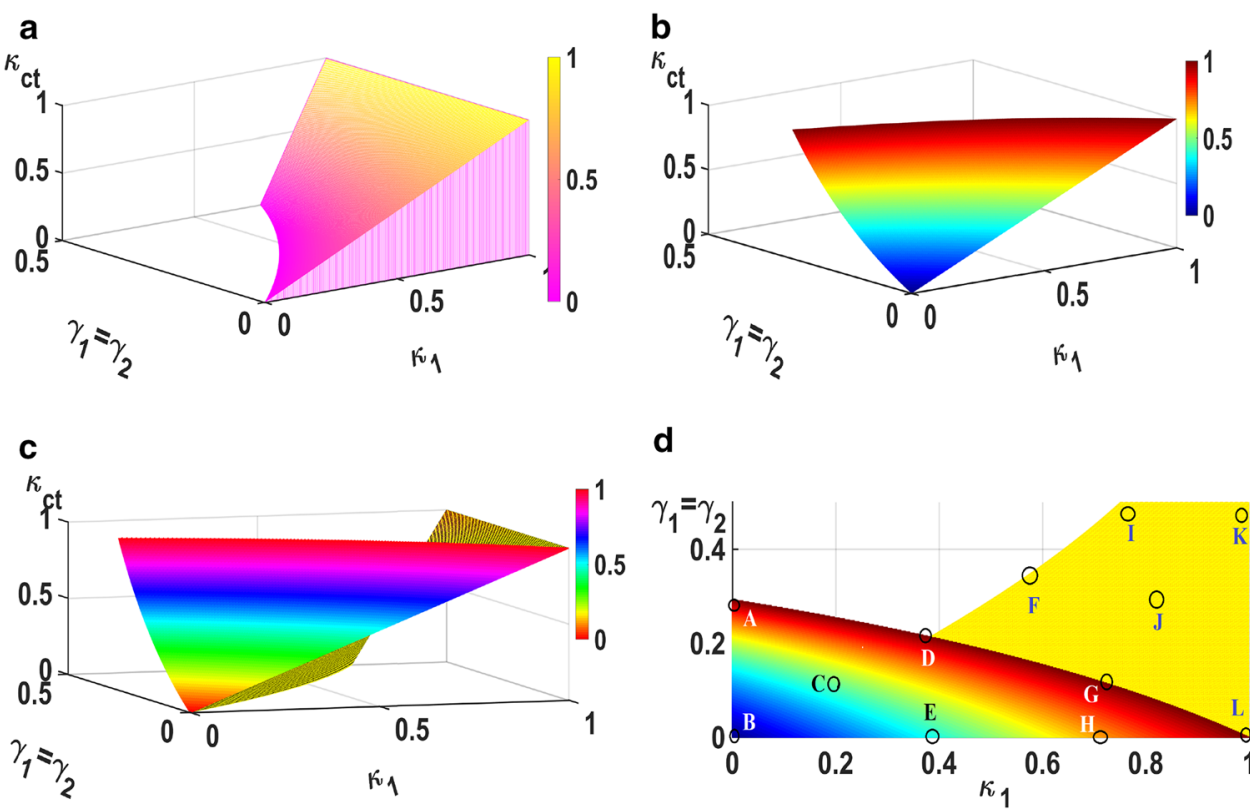

d

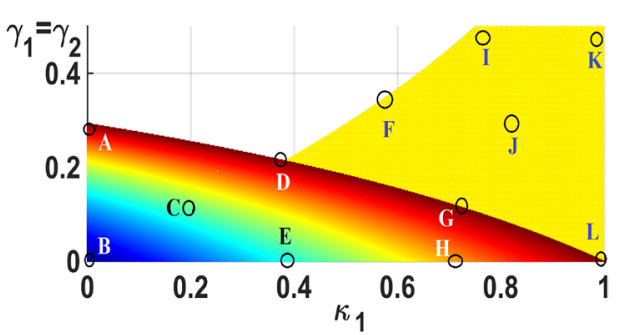

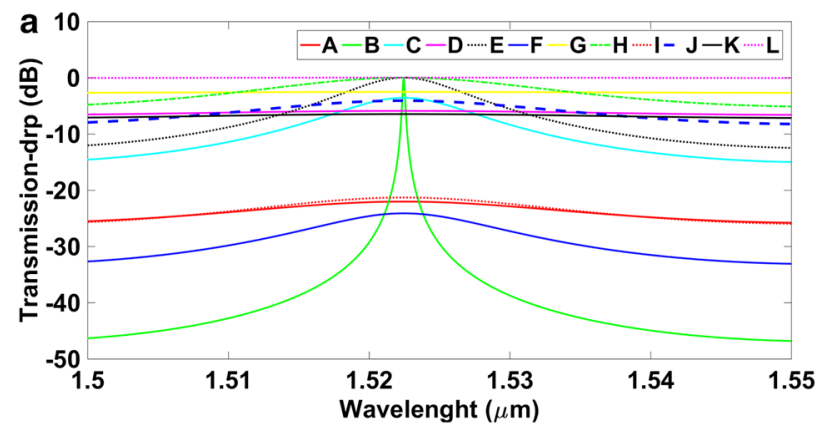
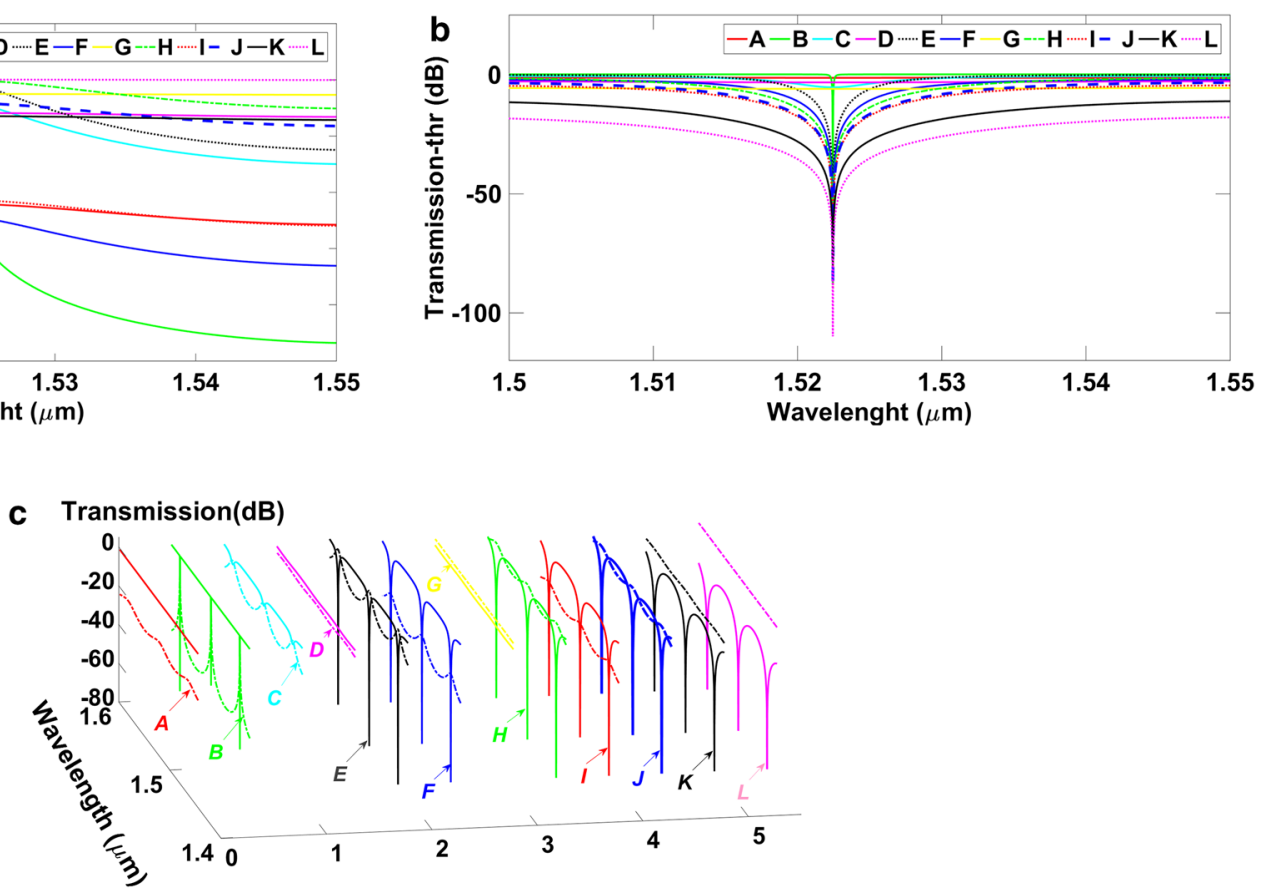

Fig. 4 Optical transmission of the add-drop resonator against wavelength for 12 selected points (A-L) from double-critical coupling condition surfaces a through port, $\mathbf{b}$ drop port, $\mathbf{c}$ through and drop ports

and $\gamma_{2}$ ) contributes to vanishing crosstalk, which can be seen for the A, D, G, K and L points in Fig. 4.

The selected points represent different transmission characteristics. The output transmission from ADR was analyzed for every selected point at resonant peak $(1.523 \mu \mathrm{m})$ by considering the bar chart of the FWHM, the $Q$-factor, the OBRR and the crosstalk for selected points. As shown in Fig. 5a, the minimum FWHM can be measured by selecting a small and lossless coupling coefficient. The optimum performance of bandpass filter can be shown by measuring the higher OBRR [41]. Among the selected points on the critical surfaces, the lossless point B possess the smallest FWHM of $0.17 \mathrm{~nm}$, the highest quality factor of 9000 and an OBRR as high as $47 \mathrm{~dB}$, which is desirable for filtering and sensing applications. Based on the definition of $Q$-factor in Eq. (9), the value of FWHM is a decisive gauge for the quality 
Table 1 The selected points from the critical coupling surfaces

\begin{tabular}{llll}
\hline Selected points & $k_{1}$ & $\gamma_{1}=\gamma_{2}$ & $k_{2}$ \\
\hline A & 0.0082 & 0.2751 & 0.9111 \\
B & 0.0090 & 0.0000 & 0.0090 \\
C & 0.2088 & 0.1084 & 0.4669 \\
D & 0.3815 & 0.2129 & 0.9955 \\
E & 0.3815 & 0.0000 & 0.3815 \\
F & 0.5221 & 0.3072 & 0.0042 \\
G & 0.7068 & 0.1205 & 0.9996 \\
H & 0.7108 & 0.0000 & 0.7108 \\
I & 0.7349 & 0.4799 & 0.0200 \\
J & 0.7631 & 0.2691 & 0.5565 \\
K & 0.9598 & 0.4940 & 0.8432 \\
L & 0.9960 & 0.0000 & 0.9960 \\
\hline
\end{tabular}

factor. As shown in Fig. 5a, almost all selected points have the FWHM as large as $10 \mathrm{~nm}$, but the FWHM for point B reaches to $0.17 \mathrm{~nm}$, which is almost 10 times less than that of from other points. It obviously brings about a $Q$-factor almost 10 times larger other points.

Large $Q$-factor is associated with low-loss resonator. These results for point B are quite compatible with the reported results in [40], which shows compatibility of
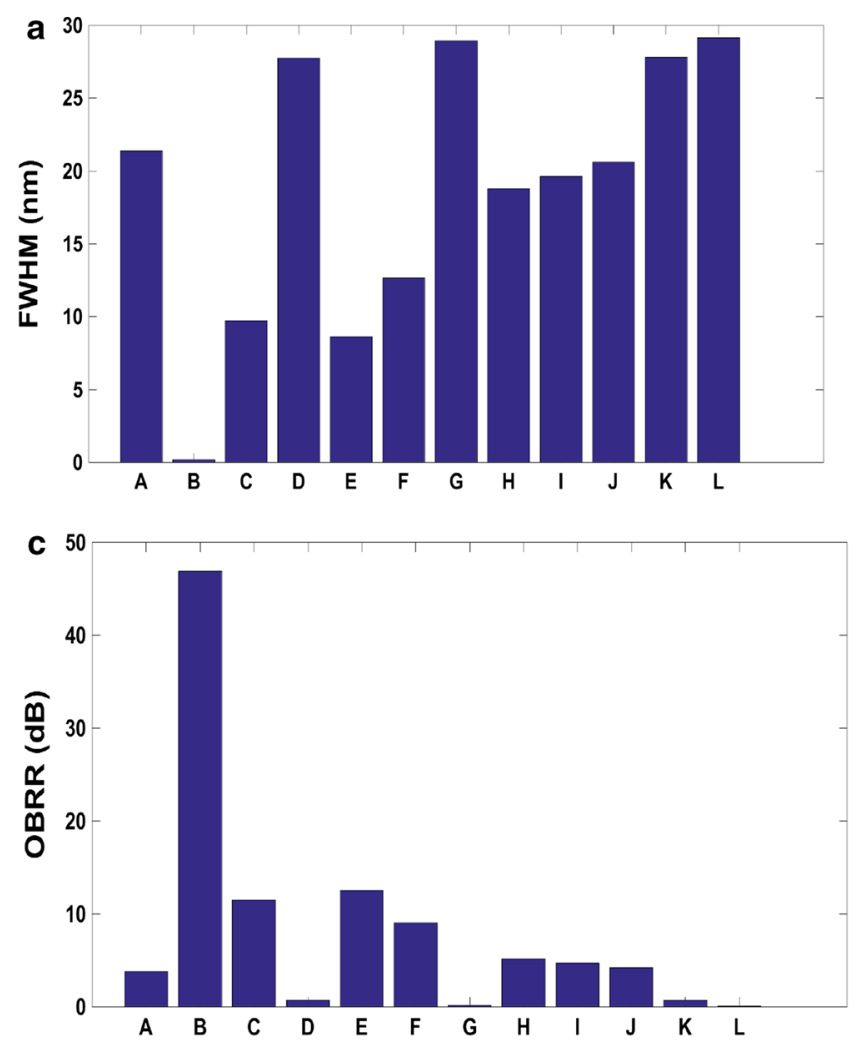

DCCC with experimental data. For the bandpass filters (here drop port of ADF), the out-of-band rejection represents the amount of applied suppression to any of the signal outside of the chosen wavelength band. The out-of-band rejection specifies a rejection value centered on resonance wavelength, which is defined in relation to a peak efficiency of a filter. In order to increase the measurement accuracy in measuring instruments, the wavelength span should be larger than out-of-band limits. According to Eq. (11), the crosstalk was defined as the difference between the maximum intensity at the drop port and the minimum intensity at the through port. As shown in Fig. 4, for some selected points located on the critical surfaces, there is no overlap between the through and drop signals. It leads to emergence of the negative values for the crosstalk. The crosstalk larger than $20 \mathrm{~dB}$ is desirable for filtering and optical communications $[20,22]$. As shown in Fig. 5d, all of the selected points fulfill the desirable crosstalk condition $(>20 \mathrm{~dB})$, but the $\mathrm{A}, \mathrm{D}, \mathrm{G}, \mathrm{K}$ and L points.

The intercept line of both critical conditions can provide the best quality for output signals. Moreover, a narrow FWHM and high OBRR can be achieved by coupling coefficient values $<0.1$ according to the results of Fig. 2 . Here, point $\mathrm{G}$ fulfills only one of the critical conditions and its coupling coefficients are larger than 0.1. As shown in Fig. 4c, there exists no overlap between the drop and the
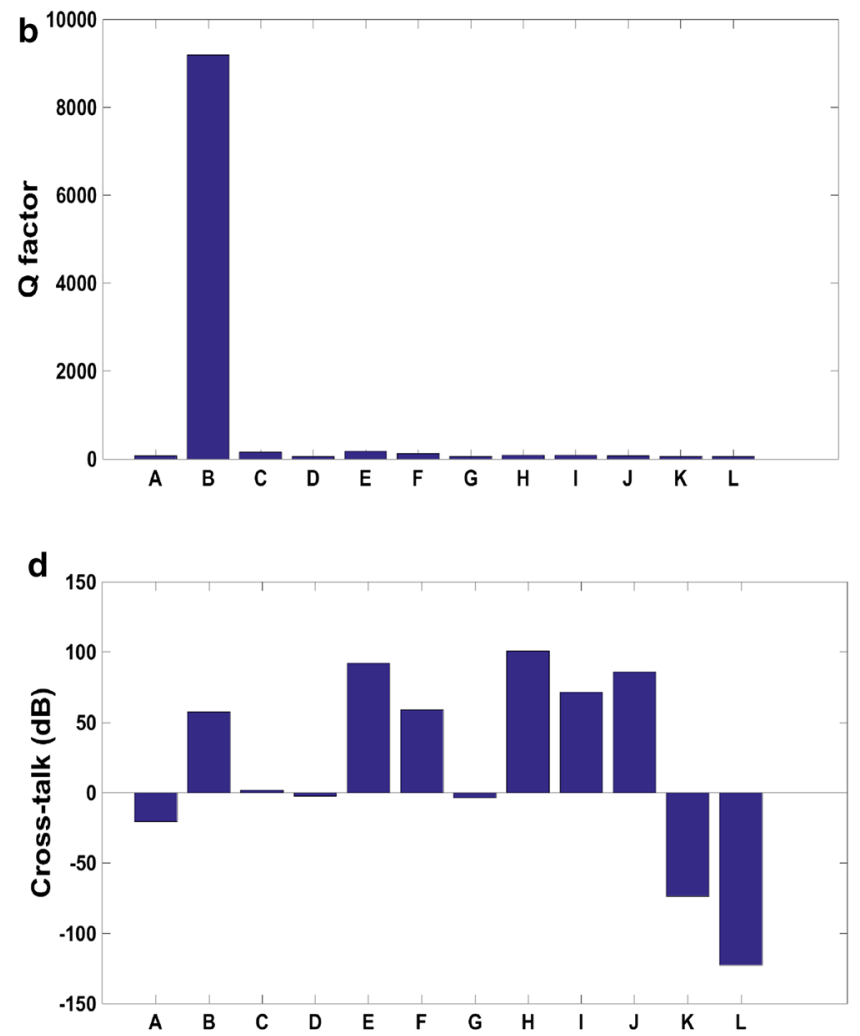

Fig. 5 The calculated optical parameters for each selected point. a The FWHM at resonant peak (1.52. $\mu$ m). b The $Q$-factor, c The OBRR and d the crosstalk 
through ports of point G. It leads to a negative crosstalk for this point as shown in Fig. 5d. The lossless points of $\mathrm{E}$ and $\mathrm{H}$ have the highest crosstalk; however, point $\mathrm{L}$ reveals that the lossless coupling is not enough condition to acquire large crosstalk and the strength of coupling coefficients is also effective parameter. These resonance features can be achieved by any add-drop resonator, which fulfills the introduced double-critical coupling condition region.

\section{Conclusion}

Double-critical coupling condition (DCCC) is introduced for lossy mode couplers. Obtaining the high-quality and tunable filtered signals from add-drop resonator was the main aim of interest. The effect of coupling loss and coupling strength on the FWHM, OBRR, $Q$-factor and crosstalk was examined. The OBRR larger than $40 \mathrm{~dB}$, the crosstalk larger than $50 \mathrm{~dB}$, highest quality factor of 9000 and the FWHM as small as $0.17 \mathrm{~nm}$ can be realized under the DCCC. The performance of add-drop resonator under DCCC reveals that the coupling strength of couplers and the amount of coupler loss should simultaneously be considered to achieve highquality output signals in practice. The introduced DCCC can determine the optimum relation between the strength of coupling coefficients and the coupling losses.

Acknowledgements IS Amiri would like to acknowledge for the research facilities of Ton Duc Thang University, Vietnam.

Open Access This article is distributed under the terms of the Creative Commons Attribution 4.0 International License (http://creativeco mmons.org/licenses/by/4.0/), which permits unrestricted use, distribution, and reproduction in any medium, provided you give appropriate credit to the original author(s) and the source, provide a link to the Creative Commons license, and indicate if changes were made.

\section{References}

1. Pointurier, Y., Brandt-Pearce, M.: Analytical study of crosstalk propagation in all-optical networks using perturbation theory. J. Lightwave Technol. 23, 4074-4083 (2005)

2. Tameh, T.A., Isfahani, B.M., Granpayeh, N., Javan, A.M.: Improving the performance of all-optical switching based on nonlinear photonic crystal microring resonators. AEU Int. J. Electron. Commun. 65, 281-287 (2011)

3. Noorden, A.F.A., Mohamad, A., Bahadoran, M., et al.: All-optical hysteresis switching using mobius configuration microring resonator circuit. Jurnal Teknologi 74, 19-25 (2015)

4. Nawi, I.N.M., Bahadoran, M., Ali, J., Yupapin, P.: A theoretical model of all-optical switching induced by a soliton pulse in nanowaveguide ring resonator. J. Phys. Conf. Ser. 431, 012029 (2013)

5. Tavousi, A.: Wavelength-division demultiplexer based on heterostructure octagonal-shape photonic crystal ring resonators. Optik 179, 1169-1179 (2019)
6. Tavousi, A., Mansouri-Birjandi, M.A., Ghadrdan, M., RanjbarTorkamani, M.: Application of photonic crystal ring resonator nonlinear response for full-optical tunable add-drop filtering. Photon Netw. Commun. 34, 131-139 (2017)

7. Bahadoran, M., Yupapin, P.: All-optical notch filters for ultrawideband chaotic communications. Eur. Phys. J. Plus. 133, 487 (2018)

8. Hagness, S., Rafizadeh, D., Ho, S., Taflove, A.: FDTD microcavity simulations: design and experimental realization of waveguide-coupled single-mode ring and whispering-gallery-mode disk resonators. J. Lightwave Technol. 15, 2154-2165 (1997)

9. Daniel, R.S., Pandeeswari, R., Raghavan, S.: Multiband monopole antenna loaded with complementary split ring resonator and C-shaped slots. AEU Int. J. Electron. Commun. 75, 8-14 (2017)

10. Mewara, H.S., Deegwal, J.K., Sharma, M.M.: A slot resonators based quintuple band-notched Y-shaped planar monopole ultrawideband antenna. AEU Int. J. Electron. Commun. 83, 470-478 (2018)

11. Rabus, D.G., Integrated Ring Resonators: The compendium. In: Integrated Ring Resonators. Springer Series in optical sciences (2007)

12. Chaiwong, K., Bahadoran, M., Amiri, I.S., et al.: Electro-optic conversion circuit incorporating a fiber optic loop for light fidelity up-down link use. Microw. Opt. Technol. Lett. 61, 526-531 (2019)

13. Bahadoran, M., Noorden, A.F.A., Mohajer, F.S., et al.: Detection of Salmonella bacterium in drinking water using microring resonator. Artif. Cells Nanomed. Biotechnol. 44, 315-321 (2016)

14. Mohamad, A., Bahadoran, M., Noorden, A., et al.: Modified adddrop microring resonator for temperature sensing. J. Comput. Theor. Nanosci. 12, 3188-3193 (2015)

15. Mohamad, A., Aziz, M., Bahadoran, M., et al.: Temperature effect on refractometric double ring resonator. Jurnal Teknologi $\mathbf{7 4}$, 35-39 (2015)

16. Yupapin, P., Saeung, P., Li, C.: Characteristics of complementary ring-resonator add/drop filters modeling by using graphical approach. Opt. Commun. 272, 81-86 (2007)

17. Vorckel, A., Monster, M., Henschel, W., Bolivar, P.H., Kurz, H.: Asymmetrically coupled silicon-on-insulator microring resonators for compact add-drop multiplexers. IEEE Photonics Technol. Lett. 15, 921-923 (2003)

18. Melloni, A., Martinelli, M.: Synthesis of direct-coupled-resonators bandpass filters for WDM systems. J. Lightwave Technol. 20, 296 (2002)

19. Dey, S., Mandal, S.: Modeling and analysis of quadruple optical ring resonator performance as optical filter using Vernier principle. Opt. Commun. 285, 439-446 (2012)

20. Mansoor, R.D., Sasse, H., Al Asadi, M., Ison, S.J., Duffy, A.P.: Over coupled ring resonator-based add/drop filters. IEEE J. Quantum Electron. 50, 598-604 (2014)

21. Chremmos, I., Schwelb, O., Uzunoglu, N.: Photonic microresonator research and applications, vol. 156. Springer, Berlin (2010)

22. Schwelb, O.: Crosstalk and bandwidth of lossy microring add/ drop multiplexers. Opt. Commun. 265, 175-179 (2006)

23. Kaalund, C.J.: Critically coupled ring resonators for add-drop filtering. Opt. Commun. 237, 357-362 (2004)

24. Oppenheim, A.V., Schafer, R.W., Buck, J.R.: Discrete-Time Signal Processing, vol. 2. Prentice hall, Englewood Cliffs (1989)

25. Sullivan, D.M.: Z-transform theory and the FDTD method. IEEE Trans. Antennas Propag. 44, 28-34 (1996)

26. Strang, G., Aarikka, K.: Introduction to Applied Mathematics, vol. 3. Wellesley-Cambridge Press, Wellesley (1986)

27. Cui, X., Gong, L., Zhao, X., Zou, H.: The Z-transform method for multidimensional dynamic economic systems. China Economics 
and Management Academy, Central University of Finance and Economics (2012)

28. Parekh, S., Gandhi, N., Hellerstein, J., et al.: Using control theory to achieve service level objectives in performance management. Real Time Syst. 23, 127-141 (2002)

29. Bahadoran, M., Ali, J., Yupapin, P.P.: Ultrafast all-optical switching using signal flow graph for PANDA resonator. Appl. Opt. 52, 2866-2873 (2013)

30. Sirawattananon, C., Bahadoran, M., Ali, J., Mitatha, S., Yupapin, P.P.: Analytical vernier effects of a PANDA ring resonator for microforce sensing application. IEEE Trans. Nanotechnol. 11, 707-712 (2012)

31. Daud, S., Chaudary, K.T., Bahadoran, M., Ali, J.: Z-transform method for optimization of add-drop configuration system. Jurnal Teknologi 74, 101-105 (2015)

32. Bahadoran, M., Yupapin, P.: Butterfly-like phase shift: a novel gauge for critical coupling of add-drop resonator. J. Theor. Appl. Phys. 12, 127-134 (2018)

33. Bahadoran, M., Noorden, A.F.A., Chaudhary, K., et al.: Nano force sensing using symmetric double stage micro resonator. Measurement 58, 215-220 (2014)

34. Bahadoran, M., Afroozeh, A., Ali, J.B., Yupapin, P.P.: Slow light generation using microring resonators for optical buffer application. Opt. Eng. 51, 044601 (2012)

35. Bahadoran, M., Ali, J., Yupapin, P.P.: Graphical approach for nonlinear optical switching by PANDA vernier filter. IEEE Photonics Technol. Lett. 25, 1470-1473 (2013)
36. Bahadoran, M., Aziz, M., Noorden, A., et al.: Novel approach to determine the Young's modulus in silicon-on-insulator waveguide using microring resonator. Digest J. Nanomater. Biostruct. 9, 1095-1104 (2014)

37. Bahadoran, M., Noorden, A., Chaudhary, K., et al.: Modeling and analysis of a microresonating biosensor for detection of Salmonella bacteria in human blood. Sensors 14, 12885-12899 (2014)

38. Vorckel, A., Monster, M., Henschel, W., Bolivar, P.H., Kurz, H.: Asymmetrically coupled silicon-on-insulator microring resonators for compact add-drop multiplexers. Photonics Technol. Lett. IEEE 15, 921-923 (2003)

39. Little, B.E., Chu, S.T., Haus, H.A., Foresi, J., Laine, J.P.: Microring resonator channel dropping filters. J. Lightwave Technol. 15, 998-1005 (1997)

40. Xu, Q., Fattal, D., Beausoleil, R.G.: Silicon microring resonators with 1.5- $\mu \mathrm{m}$ radius. Optics Express. 6, 4309-4315 (2008)

41. Little, B., Chu, S., Absil, P., et al.: Very high-order microring resonator filters for WDM applications. IEEE Photonics Technol. Lett. 16, 2263-2265 (2004)

Publisher's Note Springer Nature remains neutral with regard to jurisdictional claims in published maps and institutional affiliations. 